\title{
Computer programs in cytology reporting and record keeping
}

\author{
KV SWETTENHAM, CD NICKOLS, CL BERRY \\ From the Department of Morbid Anatomy, The London Hospital, London E1 1BB
}

SUMMARY Large numbers of specimens are handled by cytology laboratories. There is great variation in their origin, a need for a name and disease index and for statistical data about the source and nature of the specimens. A computer program was devised to reduce this workload and to improve the accuracy of record keeping. Much of the tedium of this important aspect of laboratory function can thus be removed.

We have previously described computer programs in histopathology reporting and record keeping ${ }^{1}$ pointing out the advantages in our department of this form of data handling. Others have reported different systems ${ }^{2}$ based on word processors rather than main frame systems and there is extensive documentation of area based registries using computer data storage. The various systems offer similar advantages, we believe that ours has the advantage of being cost effective as many laboratories within the Region may use the same machine and programs, greatly reducing capital expenditure, maintenance costs and programming effort.

We have devised cytology programs for reporting, record keeping and recall, the latter function now being a District function in the Health Service.

As for our previous work in histopathology, we used the North East Thames Regional Health Authority PDP-11/70 computer (via a standard British Telecom telephone link) in this program development, using Basic Plus-2 language and the file handling system provided by the Record Management Services (RMS). This supports the use of keys to unlock files for fast and accurate retrieval of specified information and facilitates the writing of programs for handling large numbers of patient specimens, with the provision of files for a name and disease index and the collection of statistical data.

\section{Methods}

We have used similar concepts in cytology to those in our histopathological programs. There are two sepa- rate file structures. The first, or main file, stores those data elements listed in Table 1 . The cytology departmental number is designated as the primary key, with the hospital record number and the patient's surname as the first and second alternates. The second, or small file, stores the data elements listed in Table 2 . The keys remain as for the first file but in addition to these three, the topographical and morphological codes are designated as third and fourth alternates,

Table 1 Map of the main file

\begin{tabular}{ll}
\hline Cytology departmental number & Primary key \\
Hospital record number & First alternate key \\
Patient's surname & Second alternate key \\
Patient's forename & \\
Consultant's initials & \\
Origin of specimen & \\
Diagnosis & \\
Specimen & \\
Date of birth/age & \\
Sex & \\
Date specimen received \\
Topographical code \\
Morphological code \\
First extra code \\
Second extra code \\
Clinical report \\
Date reported \\
Gynaecological details (yes or no) \\
Vide \\
Consultant's initials \\
Gynaecological details \\
LM P \\
Parity \\
Cervix-normal or eroded \\
Menstrual cycle \\
DXT \\
PCB \\
PMB \\
Weeks post natal \\
Type of hormone therapy \\
Total of 519 characters
\end{tabular}


Table 2 Map of the small file

Cytology departmental number
Hospital record number
Patient's sumame
Topographical code
Morphological code
First extra code
Second extra code
Patient's forename
Date of birth/age
Sex
Vide

Primary key

First alternate key

Second alternate key

Third alternate key

Fourth alternate key

Fifth alternate key

Sixth alternate key
Total of 86 characters

with two extra codes as the fifth and sixth alternates.

The main file has a provision for 519 characters per record and the small file 86 .

\section{THE MAIN FILE}

The first program allows data to be entered into the main file. These include the cytology departmental number, patient's forename and surname, the hospital record number and clinical details. The entry of " $M$ " (for male) ensures that the program by-passes the "gynaecological details" section, and "F" causes the program to ask the operator whether gynaecological details are required. Two mistake clauses allow the operator to go back and correct mistakes, a "No" entered on the final clause puts the record into the file. The program then returns to the beginning. When all the data have been entered, pressing the "RETURN" key causes the program to open the small file and search for a hospital record number to match the newly entered data. If successful, it assigns the highest cytological record number returned to the "VIDE" box in the main file. When a match has been attempted for all the newly entered data, the program ends and all files are closed.

The next program provides the laboratory with a printed daily record of specimens entered into the main file and acts as a daybook. The record includes the cytological departmental number of any previous specimen found by the automatic VIDE search program and each sheet has the date printed at the top followed by eight records.

It also can be used by the laboratory to:

(i) Provide a record of specimens to be examined.

(ii) Help in answering telephone enquiries.

(iii) Update records after having diagnosis and SNOP code entered.

The provision of a printed VIDE by this program allows easy access to all previous patient specimens.

When a specimen has been reported and coded, the new report can be entered into the main file using an update program. On typing the cytological departmental number, the VDU displays the patient's name, hospital record number and specimen allowing the operator to check that no mistake has occurred. The VDU then displays "Negative?-Type ' $N$ '". If " $N$ " is entered, the statement "no neoplastic cells seen" will be entered as the report. Otherwise using the "RETURN" key will allow the operator to enter four lines of 62 characters per line, of report. The date of reporting is then entered followed by the topographical and morphological codes and two extra codes if required. The reporting consultant's initials are added. A mistake clause is provided, allowing the operator to correct data if necessary. A negative response to the mistake query will now enter the data into the main file. The VDU then requests entry of the statistical code, this entry, plus the topographical code, is put into the statistics file and a copy of the relevant main file data is automatically transferred into the small file (see Table 2). A record of the cytological departmental number of the updated cases is stored in the print buffer file-the purpose of this is described later. When data entry is complete the use of the RETURN key causes all files to be closed and the program to end.

Up to five characters may be entered under topographical or extra codes. The morphological code permits an entry of nine characters which allow the use of the standard morphology plus a bracketed sub-classification, if required.

Reports are printed using "CYTOPT". The program gives the option of VDU display of printing. Data are printed using tractor fed paper, 8 inches wide $(20 \mathrm{~cm})$ perforated at 5.5 inches $(14 \mathrm{~cm})$, resulting in a report approximately the same size as the standard cytology form. It is possible to choose between manual or automatic printing, the latter option prints all cytology departmental numbers stored in the print buffer file. Alternatively, the operator can enter the numbers of the reports to be printed.

The display then asks for the number of copies of each report required; these are produced in the format shown in the Figure. The presence or absence of the printed box containing the gynaecological details is dictated by the information previously entered using the main file. The program will run automatically until all the numbers provided by the print buffer are used.

\section{MAIN FILE SUPPORT PROGRAMS}

The first of these supporting programs deletes data from the main file and allows corrected data to be re-entered. The second allows data to be entered or deleted in the print buffer and the third, provides the same facilities with the VIDE file. Of these, the first is used mainly to correct patient data when further, or more accurate information becomes available. The 
Table 3 Summary of functions of programs

\begin{tabular}{|c|c|}
\hline Program & Main file system \\
\hline $\begin{array}{l}\text { CYTMF } \\
\text { DAYREC } \\
\text { CUDATE }\end{array}$ & $\begin{array}{l}\text { Enters departmental number, patient details and gynaecological details where relevant and vide data. } \\
\text { A printed daily record of specimens received by the laboratory. } \\
\text { Enters report and SNOP code. Data automatically transferred to the Small file. Numbers are stored in the print } \\
\text { buffer to facilitate automatic printing and statistics are stored in the statistics file. }\end{array}$ \\
\hline $\begin{array}{l}\text { CYTOPT } \\
\text { CPBCR } \\
\text { CVCR } \\
\text { DELMF } \\
\text { CYTDEL } \\
\text { MFSEQ } \\
\text { FINDMF }\end{array}$ & $\begin{array}{l}\text { Prints the report, which is then signed by the pathologist. } \\
\text { Data entry or deletion from automatic print buffer. } \\
\text { Data entry or deletion from the automatic vide file. } \\
\text { Deletes one specified case from the main file. } \\
\text { Housekeeping program for batch deletion of reported cases. } \\
\text { Main file sequential list or only the missing cases. } \\
\text { Search and find by-departmental number, patient's surname (with or without hospital record number match) or hospital } \\
\text { record number alone. }\end{array}$ \\
\hline $\begin{array}{l}\text { CYTSF } \\
\text { CYYRUB } \\
\text { SFSEQ } \\
\text { FINDCY }\end{array}$ & $\begin{array}{l}\text { Small file system } \\
\text { Enters cytological departmental number, patient details and SNOP codes. } \\
\text { Deletes one specified case from the Small file. } \\
\text { Sequential Small file list or only the missing cases. } \\
\text { Search and find by departmental number, patients surname (with or without record number match), Hospital record } \\
\text { number alone, the topographical code, the morphological code, or both as a pair or the extra codes. }\end{array}$ \\
\hline $\begin{array}{l}\text { CYSTAT } \\
\text { EXFOCY } \\
\text { CYINST }\end{array}$ & $\begin{array}{l}\text { Statistics } \\
\text { Print out of the total number and source of specimens. } \\
\text { Exfoliative cytology print-out of specimens. } \\
\text { Data entered into or deleted from the statistics file. }\end{array}$ \\
\hline
\end{tabular}

others are used mainly to correct errors which may arise from spurious telephone noise occurring at the

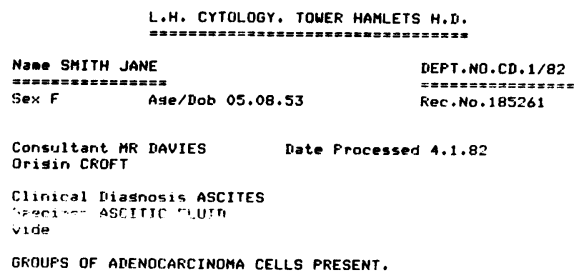
$T=Y X 01 \quad M=8146(63) \quad$ DATE 5.1 .82 SIGNED ABC
EXTRA CODES

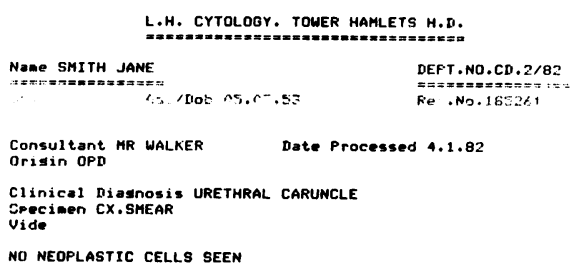

\begin{tabular}{|c|c|c|c|}
\hline $\begin{array}{l}\text { LMP } 9.11 .81 \\
\text { CX.EROSION } \\
3 / 52\end{array}$ & PARITY $2+0$ & $\begin{array}{l}\text { CYCLE } 25 / 28 \\
\text { P.NATAL O }\end{array}$ & \\
\hline $\begin{array}{l}T=8 \times 31 \quad M=0001 \\
\text { EXTRA CODES }\end{array}$ & & DATE 5.1 .82 & SIGNED ABC \\
\hline
\end{tabular}

time the information is put into the file, or printer problems.

\section{THE HOUSEKEEPING PROGRAM}

This deletes records of patient data from the main file allowing it to be reused (our main file has a blocksize of 5000 , allowing 4500 cases to be stored). The program allows data to be deleted only after checking that it has been reported and transferred into the small file. When previous cases have been checked and deletion completed the date is entered. This, and the number of the last deleted record, will be displayed the next time the program is run.

\section{MAIN FILE SEARCH AND FIND PROGRAMS}

Two programs are provided to display or print data from the main file. The first and simplest, is the sequential cytology number search. This displays or prints data between two numbers entered, or alternatively, will display, or print the missing numbers only. The second will search for data using the cytological departmental number (A), patient's surname (B), with or without a hospital record number match, or hospital record number $(C)$ alone. The use of " $A$ " will return only one case, " $B$ " or " $C$ " will return all cases found which match the entered data.

THE SMALL FILE

The function of the small file is to provide a name and disease index which can be maintained for many years. The data stored (see Table 2) comprises a total of only 86 characters per record and using a file with a 
blocksize of $\mathbf{5 0 0 0}$ should maintain six months' to a year's data for most laboratories. By changing the name of this file each year, it is a simple matter to search either the current or previous years or to search all the files for data designated by "keys" as described in the small file "Search and Find" programs.

The small file system can be used by direct entry of data or by using automatic transfer from the main file system. The program allows the operator to enter only the data required by the small file, a total of eleven elements. Alternatively, this program can be used to enter corrected data using either the small or main file system. Data can be deleted from the small file using a separate program.

THE SMALL FILE SEARCH AND FIND PROGRAMS Two programs are provided. The first gives sequential data as described for the main file. The second provides the same functions but also returns data based on topographical or morphological codes, or on the use of pairs of these codes. It will also return data using the extra code facility.

\section{THE STATISTICS PROGRAMS}

This provides two separate sets of information. The first depends on the attribution of a simple numerical code to the source of the specimen. This code enables a simple program to produce, at any defined time interval, the number of specimens that have come from any source. The second, based on the entry of the topographical code enables a simple program to provide a list of the total number of specimens of a given type that have been processed by the laboratory.

The statistics file can be used cumulatively or can be cleared and reused at specified time periods. A backup program, which will enter or delete statistical data is provided for correction purposes.

\section{Discussion}

The successful implementation of our histopathology reporting and data storage system has encouraged us to use similar techniques in cytology. We have now used the former package for three years and have found it accurate and reliable, although some changes have been made which are incorporated into these cytology programs. They have largely been aimed at saving secretarial time and include searches for the hospital record number in a buffer file as an additional way of finding previous data on the patient. There is a program adding further information or amendments to reports without complete re-entry of the previous typescript, for example, on specimens that have been decalcified or where further blocks have been taken.

Accurate record keeping and statistics are an increasingly important part of laboratory function in a time of diminishing secretarial and technical resources. Computer programs for record keeping and generation are cost effective; they do not divert expensively trained staff from essential work. Nor do they require that dull and repetitive tasks are undertaken by those under pressure. They are thus often more accurate than hasty indexing at the end of a busy day.

With regard to the practical and important points made by Briggs et al $^{2}$ regarding secretarial recruitment we have found that locums can fit more readily into the pattern of department activity in the well defined framework of this system. The drop in noise level in an office which previously contained five typewriters is also a considerable benefit.

\footnotetext{
References

' Swettenham KV, Nickols CD, Berry CL. Computer programs in histopathology record keeping. J Clin Pathol 1982;35:40-4.

2 Briggs JC, Ibrahim NB, MacKintosh I, Norris D. Practical use of a word processor in a histopathology laboratory. J Clin Pathol 1982;35:151-8.
}

Requests for reprints to: Professor CL Berry, Department of Morbid Anatomy, The London Hospital, London E1 1BB, England. 